\title{
Laboratory Diagnosis of Dengue Infection: Current Techniques and Future Strategies
}

\author{
Dinesh Subedi1 ${ }^{1}$ Andrew W. Taylor-Robinson ${ }^{*}$ \\ ${ }^{1}$ Department of Pharmacy, Little Buddha College of Health Sciences, Kathmandu, Nepal \\ ${ }^{2}$ School of Medical \& Applied Sciences, Central Queensland University, Rockhampton, Australia \\ Email: ${ }^{*}$ a.taylor-robinson@cqu.edu.au
}

Received 14 February 2014; revised 13 March 2014; accepted 20 March 2014

Copyright (C) 2014 by authors and Scientific Research Publishing Inc.

This work is licensed under the Creative Commons Attribution International License (CC BY). http://creativecommons.org/licenses/by/4.0/

(c) (i) Open Access

\begin{abstract}
Dengue is an increasingly significant vector-borne infectious disease, with over 50 million cases reported in more than half the world's recognised independent states. Dengue fever, dengue haemorrhagic fever and dengue shock syndrome are distinct clinical forms of an infection that is caused by Dengue Virus, a member of the Flaviviridae family. All four well characterized serotypes of the virus can cause the full spectrum of disease from asymptomatic infection to life-threatening symptoms. For effective prevention and/or treatment of disease symptoms, early and rapid detection of virus in specimens collected from clinically suspected persons is a requirement that remains challenging. A positive laboratory diagnosis is essential to confirm dengue virus infection and hence to inform patient therapy. Here, we consider the pros and cons of currently available methods for identification, ranging from conventional to sophisticated tests. Reports indicate the use of a variety of diagnostic methods of varying sensitivity, highlighting the necessity for standardisation and quality control. Several novel approaches are in development and demand further evaluation.
\end{abstract}

\section{Keywords}

Dengue; Dengue Fever; Flavivirus; Aedes; Vector-Borne Infectious Disease; Diagnosis; Laboratory Test

\section{Introduction}

Dengue is a vector-borne viral disease of humans that is transmitted by mosquitoes of the genus Aedes. The World Health Organization (WHO) regards dengue as a major international public health concern in tropical and

\footnotetext{
${ }^{*}$ Corresponding author.
}

How to cite this paper: Subedi, D. and Taylor-Robinson, A.W. (2014) Laboratory Diagnosis of Dengue Infection: Current Techniques and Future Strategies. Open Journal of Clinical Diagnostics, 4, 63-70. 
subtropical regions in which it is endemic. Due to a combination of population growth, unplanned urbanisation, global warming, lack of efficient mosquito control, increased air travel and insufficient public healthcare facilities, a 30-fold increase in dengue has been reported worldwide between 1960 and 2010 [1]-[3]. At present, more than 50 million cases of infection are reported annually. Of these, most people recover without any ongoing problems but 250,000 to 500,000 episodes are severe [4]. A high mortality rate in children contributes to around 25,000 deaths per year [2]. Over 110 countries of Africa, the Americas, Mediterranean, Southeast Asia and Pacific Island Nations are considered at risk [3] [5].

The causative agent of dengue is Dengue Virus (DENV), which belongs to genus Flavivirus of the family Flaviviridae. Other members of this family are responsible for a number of notable infectious diseases in humans, such as yellow fever, West Nile encephalitis, Japanese encephalitis and hepatitis C virus infection. DENV is an enveloped, positive sense RNA virus. The RNA genome contains a single open reading frame and is approximately 10.7 kilobases in length. The genome encodes a precursor polypeptide in which post-translational cleavage by host cell and virus-encoded protease results in formation of three structural proteins-capsid (C), membrane (M), and envelop (E), and seven non-structural proteins-NS1, NS2a, NS2b, NS3, NS4a, NS4b and NS5 [1] [6] [7]. These structural and non-structural proteins play several functions in the life cycle and pathogenesis of DENV. E protein, as its name suggests, is the principal surface protein of the virus particle (virion), and is involved in host cell attachment, typically to keratinocytes, the predominant cell type in human skin. E protein presents a major target for humoral immunity and mutation affects DENV virulence [7]. M protein is important for virion formation and maturation, while the nucleocapsid is formed by $\mathrm{C}$ protein. Of the nonstructural proteins, NS1 is a highly conserved glycoprotein that appears essential to pathogenesis. It is present in elevated concentrations in sera of dengue-infected patients during the early clinical phase of infection. However, detailed biological functions are yet to be ascribed. Other well characterised non-structural proteins are NS3 and NS5 which assist viral RNA synthesis and serve as an RNA-dependent RNA polymerase, respectively. There are five antigenically distinct serotypes of DENV, $1-5$, the last one of which was discovered only very recently [8]. Currently, the first four are distinguished by a plaque reduction neutralisation test. Separate serotypes frequently co-circulate in the same locality. Infection with one serotype will provide lifelong immunity to the exposed serotype but provide only transient protection against others [7] [9]-[11].

Humans are the primary host of DENV [2] [12] but it also circulates in non-human primates [13]. The virus is transmitted primarily by female Aedes mosquitoes, particularly A. aegypti and A. albopictus [1]. These species usually live between the latitudes of $35^{\circ}$ North and $35^{\circ}$ South below an elevation of 1000 metres (3300 ft) [2] [5]. They typically bite at dawn and dusk [14] [15] but may do so at any time of day throughout the year [16]. A female mosquito that takes a blood meal from a person infected with dengue fever during the initial $2-10$ day febrile period becomes itself infected with virions in the endothelial cells lining the midgut [17]. Between 8 - 10 days later, the virus spreads to other tissues including salivary glands and is subsequently released into the mosquito's saliva. Hosting the virus appears to have no cost to the arthropod vector, which remains infected for life. Cases of dengue transmission via infected blood products and organ donation have also been reported [18] [19], as has vertical transmission (from mother to child) in utero or at birth [20].

Dengue infection may be subclinical or symptomatic. Clinical illness occurs in three traditionally recognised forms: dengue fever (DF); dengue haemorrhagic fever (DHF); and dengue shock syndrome (DSS). In 2009, the WHO proposed a revised, broader-based clinical classification which is gradually being adopted: dengue; dengue with warning signs; and severe dengue [2]. DF is due to primary infection of any of five serotypes, and is generally mild and self-limited, from which recovery is complete. It is characterised by a fever for 2 - 10 days, headache, retro-orbital pain, myalgia, arthralgia and rash. DHF is due to secondary infection with a serotype different from that which caused primary infection, and is characterised by plasma leakage, thrombocytopenia and haemorrhagic manifestations along with symptoms of primary infection [2] [21]. DSS, another form of secondary infection, occurs when fluid and protein leak into the intestinal spaces and results in systemic shock. Both DHF and DSS are serious, often fatal, complications that are marked by problems of capillary permeability and disordered blood clotting. Different dengue serotypes vary in their capacity to cause severe illness but there is no clear consensus on the association between the two [22]. There is no specific therapy for dengue, such as antiviral drugs or vaccination [23], only supportive treatments for symptoms, including oral rehydration, administration of intravenous fluids and/or blood transfusion. While the majority of dengue infections are clinically inapparent, the lack of prophylaxis and poor treatment regimens for severe cases place a premium on rapid and reliable laboratory diagnosis of infection. When patients develop symptoms, they are undifferentiated and 
non-specific, which is problematic for correct clinical diagnosis. Unequivocal identification of dengue infection requires laboratory confirmation. The intent of this review is to outline the current laboratory methods for detection of dengue and to highlight areas where investigation may realise novel or improved diagnostic techniques.

\section{Laboratory Methods}

Precise laboratory diagnosis of dengue infection is important not only for appropriate, specialist clinical care but also for accurate public health surveillance. Currently, the most commonly used methods for dengue diagnosis include detection of: i) virus by cell culture; ii) viral nucleic acid; iii) DENV antigens or specific antibodies raised to them. Using a combination of two or more of these techniques increases the accuracy of diagnosis [2] [20]. Following onset of infection in patients, fever develops in synchrony with viraemia, when virus particles are found in plasma or serum, blood cells and tissues of the immune system (spleen, liver, lymph nodes, bone marrow). In this early stage, virus isolation, genome detection or antigen identification may each be used for diagnosis. However, after the acute phase has elapsed, serology is the method of choice to diagnose disease [2].

Selecting from available laboratory diagnostic methods the most appropriate technique to use in each instance depends on the purpose for which testing is performed. These include diagnosis of primary and secondary infection, analysis of data for epidemiological surveys, pathological studies and vaccine development. Moreover, demand for rapidity, cost, and availability of appropriate laboratory resources should also be considerations in choice of methodology.

\subsection{Virus Culture}

Successful isolation of virus from viraemic patients requires that samples should be collected in the early stage of disease, usually within 5 days of onset of fever [24]. Common specimens include plasma, serum, peripheral blood, cerebrospinal fluid, pleural fluid and immune system tissues [2]. Three methods of DENV cultivation have been reported, involving inoculation of specimens into mosquitoes, various in vitro cultured cell lines, and intracerebrally in mice [2] [12] [24]. Inoculation of Toxorhynchites splendens and male Aedes albopictus mosquitoes is the most sensitive method [25]. Growth of virus is confirmed by detection of antigen in crushed heads of mosquitoes by serotype-specific immunofluorescence. The rearing and handling of potentially infectious mosquitoes require excellent facilities, adherence to health and safety protocols, and stringent conditions beyond the capacity of many laboratories. As an often more practical alternative, specimens may be inoculated into any of several widely available mosquito cell lines, such as AP61, Tra-284, C6/36, AP64 and CLA-1, or into mammalian cell lines like LLCMK2, Vero and BHK21 [26]. This is less sensitive than mosquito inoculation because no wild-type dengue virus induces cytopathic effects on in vitro cell lines. Therefore, cell cultures should be screened for specific evidence of infection by immunoassay. Inoculation of mice may be an option for virus isolation if other methods are not available. Specimens can be injected intracerebrally directly into suckling mice, which develop symptoms of encephalitis. Mouse brain is used for detection of viral antigen by immunofluorescence [2]. Despite the fact that virus isolation is the gold standard for identification of dengue infection, increasingly it is being replaced by newer molecular techniques. The main reasons for this are its lengthy procedure and requirement for viability of the virus in specimens. In addition, it is not possible to differentiate between primary and secondary infection. However, a new approach is under development which reduces time needed for detection by combining reverse transcription polymerase chain reaction (RT-PCR) with culture of whole peripheral blood [27].

\subsection{Viral Nucleic Acid Detection}

Molecular techniques that are based on detection of viral nucleic acid are considered significant for the rapid identification of DENV serotypes and diagnosis of dengue infection. Common steps involved in genome detection are nucleic acid extraction, amplification and characterization of amplified products. Conventional RT-PCR, real time RT-PCR and nucleic acid sequence-based amplification (NASBA) are frequently used methods for genome detection. Major drawbacks of these techniques are potential false positive results due to sample contamination, inability to distinguish between primary and secondary infection, and relatively high cost [2] [28].

A number of methodological adaptations of the basic RT-PCR technique have been developed in recent years. Of these variants, nested RT-PCR is the most widely used in laboratories worldwide [14] [29]. This involves a 
two-step process in which primers targeting core to pre-membrane gene regions are used for initial reverse transcription and amplification steps followed by a second PCR run with serotype-specific primers [21]. The product from the second PCR has little contamination from unwanted products of primer dimers, hairpins and alternative primer target sequences. Although RT-PCR is a rapid method for detection of DENV, it becomes less sensitive and more complicated with multiple steps of amplification. Subsequently, the two-step method was modified into an "all-in-one” multiplex RT-PCR by featuring a combination of the four (now five) serotypespecific oligonucleotide primers in a single reaction tube [15]. The amplicons are analyzed by agarose gel electrophoresis and then visualised by staining with ethidium bromide dye to differentiate between serotypes.

A recent advance has seen the development of real time RT-PCR for detection of DENV in acute phase serum samples [16]. This is a one-step assay used to quantify viral RNA genome using primer pairs and probes that are specific for each dengue serotype. Amplicons are detected in "real time” in a specialised PCR machine by using chemical formats like fluorophores, 5' nuclease, adjacent linear and hairpin oligonucleotide probes and self-fluorescing amplicons. It does not require electrophoresis. Its numerous advantages over RT-PCR include rapidity, the ability to provide quantitative measurements, a low contamination rate, a higher specificity, a higher sensitivity and easy standardisation. Collectively, these improvements mean real time RT-PCR is replacing conventional RT-PCR as the new gold standard for rapid diagnosis of dengue infection with acute phase serum samples [11] [17] [30]. TaqMan ${ }^{\circledR}$ assay, one of the several types of real time RT-PCR, which uses a fluorogenic-labeled 5'-3' nuclease oligonucleotide probe, Taq DNA polymerase, is commonly used with high specificity and sensitivity. It is highly specific due to the sequence-specific hybridisation of the probe. Nevertheless, the primers and probes that have been reported previously may not be able to detect all DENV serotypes. Their sensitivity depends on homology with the targeted gene sequence of the particular virus being analysed [31]. Real time RT-PCR using the fluorescent double-stranded DNA-binding dye SYBR green ${ }^{\circledR}$ is an alternative approach that has the advantage of simplicity in primer design and uses universal RT-PCR protocols but which is less specific. Shu et al. suggested that the one-step SYBR green I-based RT-PCR system has considerable potential for deployment in clinical diagnosis and epidemiological surveillance of viral disease due to its higher sensitivity than both serology and cell culture [18]. However, further investigation is required to confirm or refute this submission.

The NABSA assay is an isothermal RNA-specific amplification technique that does not require the use of a thermal cycler. An initial reverse transcription step is followed by transcription to synthesise RNA which is detected by fluorescence. It has significant specificity and sensitivity, comparable with that of virus isolation, and may be particularly useful in field studies of dengue infection [19]. The reverse transcription loop-mediated isothermal amplification (RT-LAMP) assay is a recent modification that can be used for rapid detection of the DENV genome. This is based on the principle of a strand displacement reaction and a stem loop structure which amplifies the target to provide great specificity and selectivity. An attractive features of RT-LAMP is its ease of monitoring of amplification, which may be accomplished by SYBR green ${ }^{\circledR}$ or ethidium bromide. Moreover, RT-LAMP requires less than one hour to yield results compared to the 3 - 4 hours RT-PCR takes to run. However, performance relative to other nucleic acid amplification methods is currently not known [32].

\subsection{Serological Diagnosis}

Various methods have been developed to analyse the antigen/antibody responses during dengue infection. In comparison to other techniques available, these are generally less expensive, easier to perform and, notably, can discriminate between primary and secondary infection. However, interpretation of a serological diagnosis can be difficult since a patient may be infected with more than one serotype, anti-dengue IgG antibody can cross-react with other Flavivirus antigens, past or recent infections are hard to distinguish, and levels of IgM may be undetectably low in certain secondary infections, which thus may miss cases. Standard serological tests for DENV detection include: haemagglutination inhibition (HI); neutralisation test; indirect immunofluorescence antibody test; enzyme-linked immunosorbent assay (ELISA); complement fixation, Western blotting; and rapid immunochromatography test. Of these, HI and ELISA are the most widely used methods for routine diagnosis of dengue infection [19] [26] [33].

The HI test is a simple, cheap and rapid method of antibody detection which may be applied to large numbers of samples. Therefore, from an historical perspective it has been the most frequently used protocol for routine diagnosis of dengue. The HI test requires the analysis of paired sera collected from an individual over an interval 
of more than seven days, one during acute infection and another in the convalescent phase. Since the HI antibody persists for a long time, this is an ideal test for sero-epidemiological studies. Traditionally, it has been used to detect DENV and to differentiate primary and secondary dengue infections. As may be expected, primary infection is characterised by a slow evolution of HI antibody whereas secondary infection is notable for a rapid and elevated anamnestic response. For a positive test there may be a four-fold or greater increase in antibody titre between acute and convalescent sera. By convention, titres more than 2560 have come to represent secondary infection while those less than 1280 indicate primary infection. A major disadvantage presented by the $\mathrm{HI}$ test is the challenge to differentiate between closely related Flaviviridae members, such as dengue and Japanese encephalitis, a problem that is exacerbated by previous vaccination against Japanese encephalitis and yellow fever. Therefore, diagnosis of dengue infection by this test alone should not be considered reliable [19] [21] [34]. Moreover, HI cannot provide an early diagnosis. For this reason, the popularity of the HI test has reduced commensurate with the increased availability of more sensitive serological techniques such as ELISA.

ELISA based on immunoglobulin (Ig)M and IgG antibodies is a more efficient and popular serological test due to its simplicity, high specificity and great sensitivity. However, such antibodies can persist in the peripheral circulation for extended periods which may lead to errors in interpretation of diagnosis [2] [21]. Anti-DENV IgM is produced for a short duration during both primary and secondary infection. In primary infection, IgM rises rapidly and is usually identified after 2 - 3 days. Titres reach a peak at about 14 days, then wane gradually to undetectable levels over 2 - 3 months. Shortly after this time anti-DENV IgG appears. In secondary infection, IgM concentrations are maximal around 2 weeks after acute infection and then diminish to negligibility by 4 weeks. Unlike for primary infection, IgG appears quite rapidly, sometimes earlier than the IgM response [26] [35]. In both primary and secondary dengue infection, a rise in DENV E/M protein-specific IgM can be measured by IgM antibody capture (MAC). This has a slightly greater sensitivity than HI using paired sera for diagnosis of dengue in an individual. However, at present it is not possible to use MAC ELISA to identify dengue serotype as antibodies to DENV 1 - 4 (and most probably DENV 5) are highly cross-reactive. Recently, a MAC ELISA that distinguishes serotype has been proposed but this requires further evaluation [36]. IgG E/M-specific antibody capture (GAC) ELISA enables measurement of IgG antibodies for up to 10 months after infection, thereby facilitating detection of recent and past dengue infection. An indirect IgG ELISA has also been developed that is comparable to the HI test and likewise can be used to differentiate primary from secondary dengue infection [37]. As for other serological tests, a four-fold or greater rise in dengue-specific IgG in acute and convalescent paired sera is indicative of recent infection [2]. It is recommended that analysis of paired serum samples from acute and convalescent phases should be performed by both MAC-ELISA and IgG ELISA in order to provide confirmation of results [21]. IgM/IgG ratio is often used to discriminate primary and secondary dengue infection. If the ratio of IgM to IgG, as measured by titre, is more than 1.2, the serum sample is considered as taken from primary infection, while an IgM/IgG ratio of less than 1.2 is indicative of secondary infection [2] [38]. The IgG avidity test, a newer approach, has proved to be beneficial for determining primary and secondary dengue infections. IgG avidity index, which requires only a single sample of acute phase serum, is used to characterise dengue infection. While it shows promise, however, this test is currently not readily accessible globally [39]. A number of commercial test kits that claim rapid detection of anti-dengue IgM and IgG have been marketed in the past few years [40]. Unfortunately, the accuracy of some of these ready-made kits has either been questioned, is unknown because proper validation studies have not been performed or the results not made publically available [41]-[43].

DENV NS1 antigen has gained considerable interest as a new biomarker for early diagnosis of dengue infection [44]-[46]. NS1 antigen is abundant in serum of patients during the early stage of infection [47]. It can be detected in peripheral blood before the formation of antibodies, from the first day after onset of fever up to day 9. Studies revealed that the detection rate of NS1 antigen is higher in acute primary infection than in acute secondary infection [10]. All serotype-specific NS1 assays are highly specific for detection and determination of the appropriate serotype [44]. Hence, assay for NS1 antigen provides a potential means for the early diagnosis of dengue infection [46].

\section{Conclusions}

Diagnostic methodologies for the identification of dengue that are based on the detection of DENV-specific antibodies are considered the mainstay of clinical diagnosis of this important infectious disease. These techniques are widely employed because they are convenient to use, provide rapid results, are reasonably accurate, and are 
cheap to perform. The traditional HI test has been largely replaced by ELISA based on detection of IgM and IgG because of its reported high degree of specificity and sensitivity. However, some contradictory findings suggest the need for supplementary assessment. Detection of NS1 antigen and antibodies specific to it is a novel serological strategy that is showing promise for rapid identification. Further development of this assay should focus on the feasibility of its use in small-scale laboratories and at reduced expense.

The last decade has seen a rapid and considerable improvement in molecular methods of diagnosis. Due to their high sensitivity, tests founded on molecular strategies are looking to replace virus isolation, which is a lengthy and labour-intensive process, as the gold standard method of dengue detection. Real time RT-PCR based on TaqMan ${ }^{\circledR}$ or SYBR green ${ }^{\circledR}$, NABSA assay and RT-LAMP are recent advances in molecular techniques, each of which has a high degree of specificity and sensitivity comparable to that of virus isolation. However, a requirement for quality assurance demands further evaluation and comparison with the most sensitive methods that are currently available.

\section{Acknowledgements}

The authors' research is supported by the Central Queensland University Health CRN and the Australian Government's Collaborative Research Networks Program.

The authors have declared no competing interests.

\section{References}

[1] Gubler, D.J. (1998) Dengue and Dengue Hemorrhagic Fever. Clinical Microbiology Reviews, 11, 480-496.

[2] World Health Organization (2009) Dengue-Guidelines for Diagnosis, Treatment, Prevention and Control. New Edition, World Health Organization, Geneva, Switzerland.

[3] Guzman, M.G., Halstead, S.B., Artsob, H., Buchy, P., Farrar, J., et al. (2010) Dengue: A Continuing Global Threat. Nature Reviews Microbiology, 8, S7-S16. http://dx.doi.org/10.1038/nrmicro2460

[4] Gibbons, R.V. and Vaughn, D.W. (2002) Dengue: An Escalating Problem. British Medical Journal, 324, $1563-1566$. http://dx.doi.org/10.1136/bmj.324.7353.1563

[5] Guha-Sapir, D. and Schimmer, B. (2005) Dengue Fever: New Paradigms for a Changing Epidemiology. Emerging Themes in Epidemiology, 2, 1-10. http://dx.doi.org/10.1186/1742-7622-2-1

[6] Dussart, P., Labeau, B., Lagathu, G., Louis, P., Nunes, M.R.T., et al. (2006) Evaluation of an Enzyme Linked Immunoassay for Detection of Dengue Virus NS1 Antigen in Human Serum. Clinical and Vaccine Immunology, 13, 11851189. http://dx.doi.org/10.1128/CVI.00229-06

[7] Burke, D.S. and Monath, T.P. (2001) Flavivirus. In: Knipe, D.M. and Howley, P.M., Eds., Field Virology, 4th Edition, Lippincott Williams \& Wilkins, Philadelphia, 852-921.

[8] Normile, D. (2013) Surprising New Dengue Virus Throws a Spanner in Disease Control Efforts. Science, $342,415$. http://dx.doi.org/10.1126/science.342.6157.415

[9] Alcon, S., Talarmin, A., Debruyne, M., Falconar, A., Deubel, V., et al. (2002) Enzyme-Linked Immunosorbent Assay Specific to Dengue Virus Type 1 Nonstructural Protein NS1 Reveals Circulation of the Antigen in the Blood during the Acute Phase of Disease in Patients Experiencing Primary or Secondary Infections. Journal of Clinical Microbiology, 40, 376-381. http://dx.doi.org/10.1128/JCM.40.02.376-381.2002

[10] Young, P.R., Hilditch, P.A., Bletchly, C. and Halloran, W. (2000) An Antigen Capture Enzyme-Linked Immunosorbent Assay Reveals High Levels of Dengue Virus Protein NS1 in the Sera of Infected Patients. Journal of Clinical Microbiology, 38, 1053-1057.

[11] Leitmeyer, K.C., Vaughn, D.W., Watts, D.M., Salas, R., Chacon, I.V., et al. (1999) Dengue Virus Structural Differences That Correlate with Pathogenesis. Journal of Virology, 73, 4738-4747.

[12] Kuberski, T. and Rosen, L. (1977) A Simple Technique for the Detection of Dengue Antigen in Mosquitoes by Immunofluorescence. American Journal of Tropical Medicine and Hygiene, 26, 533-537.

[13] Chen, R.F., Yeh, W.T., Yang, M.Y. and Yang, K.D. (2001) A Model of the Real-Time Correlation of Viral Titers with Immune Reactions in Antibody Dependent Enhancement of Dengue-2 Infections. FEMS Immunology \& Medical Microbiology, 30, 1-7. http://dx.doi.org/10.1111/j.1574-695X.2001.tb01542.x

[14] Sangasang, A., Wibulwattanakij, S., Chanama, S., Orapinpatipat, A., Anegoonpipat, A., et al. (2003) Evaluation of RT-PCR as a Tool for Diagnosis of Secondary Dengue Virus Infection. Japanese Journal of Infectious Diseases, 56, 205-209.

[15] Harris, E., Roberts, T.G., Smith, L., Selle, J., Kramer, L.D., et al. (1998) Typing of Dengue Viruses in Clinical Speci- 
mens and Mosquitoes by Single Tube Multiplex Reverse Transcriptase PCR. Journal of Clinical Microbiology, 36, 2634-2639.

[16] Callahan, J.D., Wu, S.J., Dion-Schultz, A., Mangold, B.E., Peruski, L.F., et al.(2001) Development and Evaluation of Serotype and Group-Specific Fluorogenic Reverse Transcriptase PCR (TaqMan) Assays for Dengue Virus. Journal of Clinical Microbiology, 39, 4119-4124. http://dx.doi.org/10.1128/JCM.39.11.4119-4124.2001

[17] Wang, W.K., Sung, T.L., Tsai, Y.C., Kao, C.L., Chang, S.M., et al. (2002) Detection of Dengue Virus Replication in Peripheral Blood Mononuclear Cells from Dengue Virus Type 2-Infected Patients by a Reverse TranscriptionReal-Time PCR Assay. Journal of Clinical Microbiology, 40, 4472-4478. http://dx.doi.org/10.1128/JCM.40.12.4472-4478.2002

[18] Shu, P.Y., Chang, S.F., Kuo, Y.C., Yueh, Y., Chien, L.J., et al. (2003) Development of Group- and Serotype-Specific One-Step SYBR Green I-Based Real-Time Reverse Transcription-PCR Assay for Dengue Virus. Journal of Clinical Microbiology, 41, 2408-2416. http://dx.doi.org/10.1128/JCM.41.6.2408-2416.2003

[19] Wu, S.J., Lee, E.M., Putvatana, R., Shurtliff, R.N., Porter, K.R., et al. (2001) Detection of Dengue Viral RNA Using a Nucleic Acid Sequence-Based Amplification Assay. Journal of Clinical Microbiology, 39, 2794-2798. http://dx.doi.org/10.1128/JCM.39.8.2794-2798.2001

[20] Gubler, D.J. and Sather, G.E. (1988) Laboratory Diagnosis of Dengue and Dengue Hemorrhagic Fever. In: Homma, A. and Cunha, J. F., Eds., Proceedings of the International Symposium on Yellow Fever and Dengue, Bio-Manguinhos, Rio de Janeiro, 291-322.

[21] Shu, P.Y. and Huang, J.H. (2004) Current Advances in Dengue Diagnosis. Clinical and Diagnostic Laboratory Immunology, 11, 642-650.

[22] Endy, T.P., Chunsuttiwat, S., Nisalak, A., Libraty, D.H., Green, S., Rothman, A.L., Vaughn, D.W. and Ennis, F.A. (2002) Epidemiology of Inapparent and Symptomatic Acute Dengue Virus Infection: A Prospective Study of Primary School Children in Kamphaeng Phet, Thailand. American Journal of Epidemiology, 156, 40-51. http://dx.doi.org/10.1093/aje/kwf005

[23] Perng, G.C., Lei, H.Y., Lin, Y.S. and Chokephaibulkit, K. (2011) Dengue Vaccines: Challenge and Confrontation. World Journal of Vaccines, 1, 109-130.

[24] Lam, S.K. (1986) Isolation of Dengue Viruses by Intracerebral Inoculation of Mosquito Larvae. Journal of Virological Methods, 14, 133-140. http://dx.doi.org/10.1016/0166-0934(86)90044-3

[25] Win, T. (1982) Detection of Dengue Viruses by Immunofluorescence of the Intracerebral Inoculation of Mosquitoes. Lancet, i, 53-54.

[26] Guzman, M.G. and Kouri, G. (1996) Advances in Dengue Diagnosis. Clinical and Diagnostic Laboratory Immunology, 3, 621-627.

[27] Klungthong, C., Gibbons, R.V., Thaisomboonsuk, B., Nisalak, A., Kalayanarooj, S., Thirawuth, V., Nutkumhang, N., Mammen Jr., M.P. and Jarman, R.G. (2007) Dengue Virus Detection Using Whole Blood for Reverse Transcriptase PCR and Virus Isolation. Journal of Clinical Microbiology, 45, 2480-2485. http://dx.doi.org/10.1128/JCM.00305-07

[28] Deubel, V. (1997) The Contribution of Molecular Techniques to the Diagnosis of Dengue Infection. In: Gubler, D.J. and Kuno, G., Eds., Dengue and Dengue Hemorrhagic Fever, CAB International, London, 335-366.

[29] Lanciotti, R.S., Calisher, C.H., Gubler, D.J., Chang, G.J. and Vorndam, A.V. (1992) Rapid Detection and Typing of Dengue Viruses from Clinical Samples by Using Reverse Transcriptase-Polymerase Chain Reaction. Journal of Clinical Microbiology, 30, 545-551.

[30] Houng, H.H., Chen, R.C.M., Vaughn, D.W. and Kanesa-Thasan, N. (2001) Development of a Fluorogenic RT-PCR System for Quantitative Identification of Dengue Virus Serotypes 1-4 Using Conserved and Serotype-Specific 3 Noncoding Sequences. Journal of Virological Methods, 95, 19-32. http://dx.doi.org/10.1016/S0166-0934(01)00280-4

[31] Laue, T., Emmerich, P. and Schmitz, H. (1999) Detection of Dengue Virus RNA in Patients after Primary or Secondary Dengue Infection by Using the TaqMan Automated Amplification System. Journal of Clinical Microbiology, 37, 25432547.

[32] Parida, M., Horioke, K., Ishida, H., Dash, P.K., Saxena, P., Jana, A.M., Islam, M.A., Inoue, S., Hosaka, N. and Morita, K. (2005) Rapid Detection and Differentiation of Dengue Virus Serotype by a Real Time Reverse Transcription-Loop Mediated Isothermal Amplification Assay. Journal of Clinical Microbiology, 43, 2895-2903. http://dx.doi.org/10.1128/JCM.43.6.2895-2903.2005

[33] Innis, B.L., Nisalak, A., Nimmannitya, S., Kusalerdchariya, S., Chongswasdi, V., Suntayakorn, S., Puttisri, P. and Hoke, C.H. (1989) An Enzyme-Linked Immunosorbent Assay to Characterize Dengue Infections Where Dengue and Japanese Encephalitis Co-Circulate. American Journal of Tropical Medicine and Hygiene, 40, 418-427.

[34] Clarke D.H. and Casals, J. (1958) Techniques for Hemagglutination and Hemagglutination-Inhibition with ArthropodBorne Viruses. American Journal of Tropical Medicine and Hygiene, 7, 561-573. 
[35] Burke, D.S., Nisalak, A. and Ussery, M.A. (1982) Antibody Capture Immunoassay Detection of Japanese Encephalitis Virus Immunoglobulin M and G Antibodies in Cerebrospinal Fluid. Journal of Clinical Microbiology, 16, 1034-1042.

[36] Vazquez, S., Perez, A.B., Ruiz, D., Rodriguez, R., Pupo, M., Calzada, N., González, L., González, D., Castro, O., Serrano, T. and Guzmán, M.G. (2005) Serological Markers during Dengue 3 Primary and Secondary Infections. Journal of Clinical Virology, 33, 132-137. http://dx.doi.org/10.1016/j.jcv.2004.10.013

[37] Chungue, E., Marche, R., Plichart, R., Boutin, J.P. and Roux, J. (1989) Comparison of Immunoglobulin G EnzymeLinked Immunosorbent Assay (IgG-ELISA) and Hemagglutination Inhibition (HI) Test for the Detection of Dengue Antibodies. Prevalence of Dengue IgG-ELISA Antibodies in Tahiti. Transactions of the Royal Society of Tropical Medicine and Hygiene, 83, 708-771. http://dx.doi.org/10.1016/0035-9203(89)90404-5

[38] Shu, P.Y., Chen, L.K., Chang, S.F., Sue, C.L., Chien, L.J., Chin, C., Lin, T.H. and Huang, J.H. (2004) Dengue Virus Serotyping Based on Envelope/Membrane (E/M) and Nonstructural Protein NS1 Serotype-Specific Capture Immunoglobulin M (IgM) Enzyme-Linked Immunosorbent Assays (ELISA). Journal of Clinical Microbiology, 42, $2489-2494$. http://dx.doi.org/10.1128/JCM.42.6.2489-2494.2004

[39] Matheus, S., Deparis, X., Labeau, B., Lelarge, J., Morvan, J. and Dussart, P. (2005) Use of Four Dengue Virus Antigens for Determination of Dengue Immune Status by Enzyme-Linked Immunosorbent Assay of Immunoglobulin G Avidity. Journal of Clinical Microbiology, 43, 5784-5786. http://dx.doi.org/10.1128/JCM.43.11.5784-5786.2005

[40] Groen, J., Koraka, P., Velzing, J., Copra, C. and Osterhaus, A.D. (2000) Evaluation of Six Immunoassays for Detection of Dengue Virus-Specific Immunoglobulin M and G Antibodies. Clinical and Diagnostic Laboratory Immunology, 7, 867-871.

[41] Nunes, M.R., Neto, J.P., Casseb, S.M., Nunes, K.N., Martins, L.C., et al. (2011) Evaluation of an Immunoglobulin MSpecific Capture Enzyme-Linked Immunosorbent Assay for Rapid Diagnosis of Dengue Infection. Journal of Virological Methods, 17, 13-20. http://dx.doi.org/10.1016/j.jviromet.2010.09.021

[42] Aryati, A., Trimarsanto, H., Yohan, B., Wardhani, P., Fahri, S. and Sasmono, R.T. (2013) Performance of Commercial Dengue NS1 ELISA and Molecular Analysis of NS1 Gene of Dengue Viruses Obtained during Surveillance in Indonesia. BMC Infectious Diseases, 13, 611. http://dx.doi.org/10.1186/1471-2334-13-611

[43] Ho, T.S., Wang, S.M., Lin, Y.S. and Liu, C.C. (2013) Clinical and Laboratory Predictive Markers for Acute Dengue Infection. Journal of Biomedical Science, 20, 75. http://dx.doi.org/10.1186/1423-0127-20-75

[44] Ding, X., Hu, D., Chen, Y., Di, B., Jin, J., Pan, Y., Qiu, L., Wang, Y., Wen, K., Wang, M. and Che, X. (2011) Full Serotype- and Group-Specific NS1 Capture Enzyme-Linked Immunosorbent Assay for Rapid Differential Diagnosis of Dengue Virus Infection. Clinical and Vaccine Immunology, 18, 430-434.

[45] Kassim, F.M., Izati, M.N., TgRogayah, T.A., Apandi, Y.M. and Saat, Z. (2011) Use of Dengue NS1 Antigen for Early Diagnosis of Dengue Virus Infection. Southeast Asian Journal of Tropical Medicine and Public Health, 42, 562-569.

[46] Sanyal, S. and Taylor-Robinson, A.W. (2013) Host-Virus Interactions in Dengue Infection Indicate Targets for Detection and Therapeutic Interventions. Immunity and Diseases, 1, a7 1-4.

[47] Kumarasamy, V., Wahab, A.H., Chua, S.K., Hassan, Z., Chem, Y.K., Mohamada, M. and Chua, K.B. (2007) Evaluation of a Commercial Dengue NS1 Antigen-Capture ELISA for Laboratory Diagnosis of Acute Dengue Virus Infection. Journal of Virological Methods, 140, 75-79. http://dx.doi.org/10.1016/j.jviromet.2006.11.001 